\title{
METHOD OF CALCULATING MAGNETIC SYSTEM USING FINITE DIFFERENCE METHOD
}

\author{
Gennady Nikitenko, Evgeny Konoplev, Vladimir Salpagarov, Pavel Konoplev, Andrey Bobryshev \\ Stavropol State Agrarian University, Russia \\ nikitenko_gv@mail.ru, konoplev82@mail.ru
}

\begin{abstract}
The article presents a method for calculating the magnetic system using the finite difference method for the axisymmetric system. The finite difference method for constructing a model of the generator's magnetic field allows integrating differential equations into partial derivatives by reducing the equations in finite differences. A special feature of the presented calculation method is the automation of determining the boundaries of the media interface, by taking into account the magnetic resistance of adjacent nodes relative to the calculated node. The main characteristic of a magnetic field is magnetic induction, which is a vector quantity. Magnetic induction is calculated based on the distribution pattern of the magnetic potential. Based on the Poisson and Laplace equations presented in the article, a computer program was developed for automating the calculation of the magnetic induction vector. The results of the program for calculating the magnetic field pattern on the example of a synchronous generator with a two-circuit magnetic system used as a part of a wind generator are presented. Graphical dependences of the change in the value of magnetic induction inside the stator coil of a synchronous generator are constructed for a rotor with an external magnetic circuit, for a rotor with an internal magnetic circuit, and for a rotor with two magnetic circuits simultaneously. In the calculation of the magnetic system, as presented in article, by the method of the example synchronous generator with dual magnetic system, the maximum value of magnetic induction, penetrating the winding of the synchronous generator along the axis $r$, when the internal circuit is $0.21 \mathrm{~T}$, using the external loop, the value of the magnetic induction vector is 0.283 $\mathrm{T}$. When using a two-circuit system, the value of the magnetic induction vector is $0.483 \mathrm{~T}$.
\end{abstract}

Keywords: modeling; magnetic field; generator; power supply; wind power.

\section{Introduction}

Let us consider the method of calculating the magnetic system by the finite difference method on the example of a synchronous generator with a two-circuit magnetic system $[1 ; 2]$. The finite difference method for constructing a model of the generator magnetic field allows integrating differential equations into partial derivatives by reducing the equations in finite differences [3-6].

The main problem that arises when calculating magnetic fields by this method, is the inability to account for the boundaries of media of different materials, so this article offers an improvement of this calculation method, which allows to determine the boundaries of media of materials, which allows to automate the calculation process. The synchronous generator under study has: an axis of symmetry, a magnetic system, a winding, permanent neodymium magnets, electric and magnetic fields. The main characteristic of the magnetic field is the magnetic induction In, which is a vector quantity. Magnetic induction is calculated based on the distribution pattern of the magnetic potential.

The calculation of a synchronous generator with a two-circuit magnetic system is based on a vector mathematical model. The advantage of the finite difference method is the ability to create a computer program for calculating magnetic fields. Let us present a scheme for calculating the synchronous generator sector in Figure 1. The grid step on $r-0.5 \mathrm{~mm}$, on $z-1^{\circ}$.

As shown in Figure 1, we calculate the distribution of the magnetic potential vector of a synchronous generator, while for the axisymmetric case, the angle changes from $-25^{\circ}$ to $25^{\circ}$ along the $\mathrm{Z}$ axis, and all elements along the $\mathrm{R}$ axis of the magnetic system are located in the coordinates from 103 to $180 \mathrm{~mm}$ along the $\mathrm{R}$ axis.

\section{Materials and methods}

The specific magnetic resistance of a material is determined from expression (1).

$$
v=\frac{1}{\mu_{0} \mu}
$$

where $\mu_{0}$ - magnetic permeability of vacuum;

$\mu$ - relative magnetic permeability of a substance. 
A permanent magnet can be described by specifying the coercive force. The coercive force is considered as a piecewise constant function of coordinates. Its contribution to the equation is equivalent to surface currents flowing along the boundaries of permanent magnets. The effective current flowing along the upper boundary of the magnet is numerically equal to $H_{c}$, and on the lower border of the magnet $-H_{c}$.

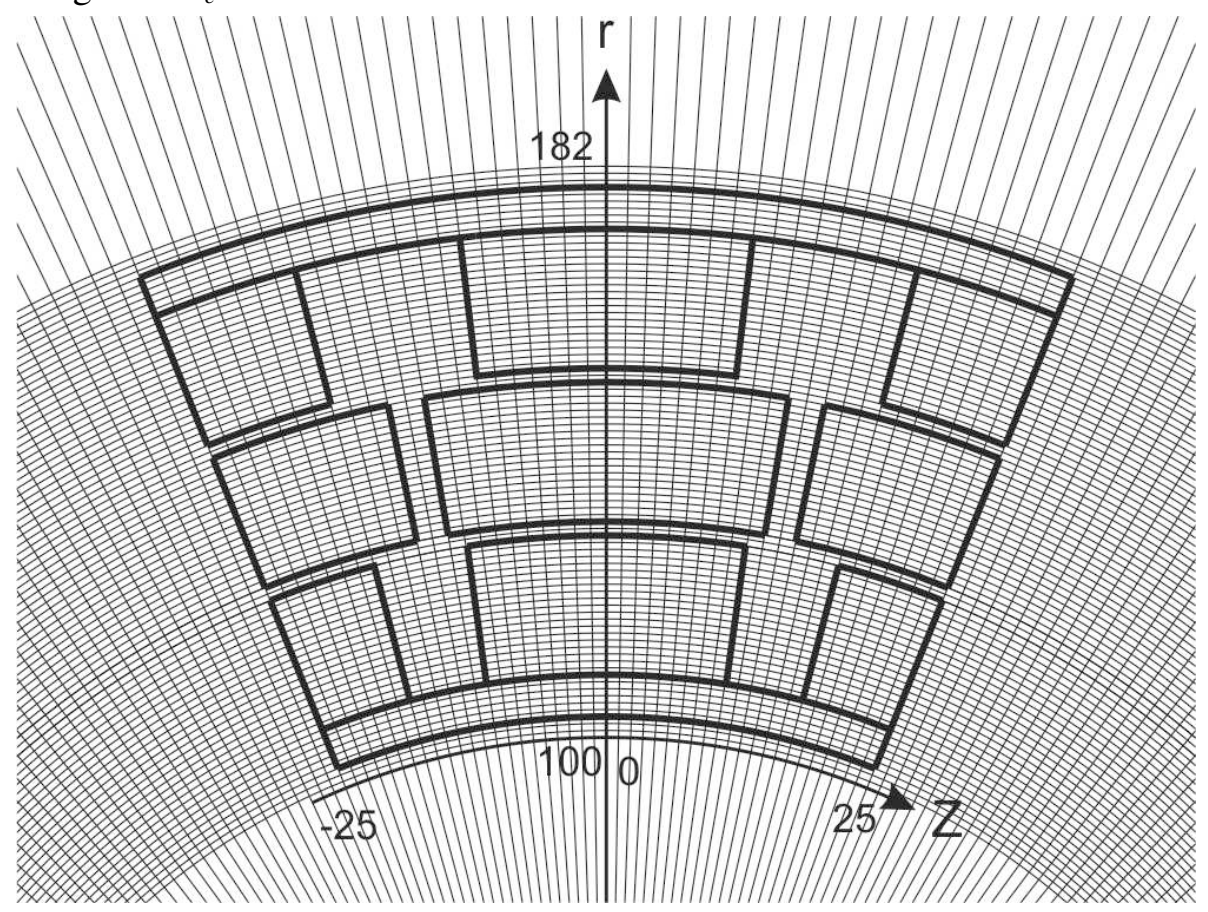

Fig. 1. Grid on the synchronous generator sector

The Poisson equation is represented by expression (2).

$$
\frac{\partial}{\partial r}\left(v \frac{1}{r} \frac{\partial(r A)}{\partial r}\right)+\frac{\partial}{\partial z}\left(v \frac{\partial A}{\partial z}\right)=j+\left(\frac{\partial H_{c r}}{\partial z}+\frac{\partial H_{c z}}{\partial r}\right)
$$

where $r, z$-coordinates of the axisymmetric model;

$A$ - magnetic potential;

$H_{c}$ - coercive force of a permanent magnet;

$j$ - current density in the conductor. (3).

The current density is a constant value in the conductor block and is determined from expression

$$
\begin{gathered}
j=j_{s t}+j_{v} \\
j_{s t}=g \cdot \operatorname{grad} U \\
j_{v}=g \frac{\partial A}{\partial t}
\end{gathered}
$$

where $j_{s t}-$ third-party conductor current, A;

$j_{v}$ - eddy current, A;

$g$ - electrical conductivity of the material, $\mathrm{Cmm}^{-1}$;

$U$ - electrical potential applied to the conductor, $\mathrm{V}$.

The synchronous generator is an axisymmetric system, therefore, to calculate the electromagnetic field, it is necessary to use the Poisson and Laplace equations in cylindrical coordinates.

$$
\frac{1}{r} \frac{\partial}{\partial r}\left(r \frac{\partial A}{\partial r}\right)+\frac{\partial^{2} A}{\partial z^{2}}=\mu \mu_{0} J
$$




$$
\begin{gathered}
\frac{1}{r} \frac{\partial}{\partial r}\left(r \frac{\partial A}{\partial r}\right)+\frac{\partial^{2} A}{\partial z^{2}}=\mu \mu_{0}\left(\frac{\partial H_{c r}}{\partial z}+\frac{\partial H_{c z}}{\partial r}\right) \\
\frac{1}{r} \frac{\partial}{\partial r}\left(r \frac{\partial A}{\partial r}\right)+\frac{\partial^{2} A}{\partial z^{2}}=0
\end{gathered}
$$

Expression (6) is used for calculating the magnetizing coil, (7) - for calculating permanent magnets, (8) - for calculating the magnetic core and non-current-carrying parts.

When using the theory of finite differences, it is sufficient to replace the field equations with a system of equations in finite differences that connect the values of the potential function A at discrete points.

The application of the difference potential method begins with the overlay of the grid on the study area, as shown in Figure 1. The study area is covered by a grid in $r$ increments $h_{r}=\left|r_{2}-r_{1}\right|$, by $z$ in increments $h_{z}=r \cdot \sin \left(\left|\varphi_{2}-\varphi_{1}\right|\right)$. The grid step along the $\mathrm{z}$ axis is determined by the cosine of the rotation angle module $\left|\varphi_{2}-\varphi_{1}\right|$ values $r$.

In the Poisson and Laplace formulas, partial derivatives are replaced by finite-difference approximations from the Taylor equation, as a result, we obtain the following expressions:

$$
\begin{aligned}
& \frac{\partial^{2} A}{\partial r^{2}} \mathrm{I}_{r, z} \approx \frac{A_{r+1, z}-2 A_{r, z}+A_{r-1, z}}{h_{r} h_{z}} \\
& \frac{\partial^{2} A}{\partial z^{2}} \mathrm{I}_{r, z} \approx \frac{A_{r, z+1}-2 A_{r, z}+A_{r, z-1}}{h_{r} h_{z}}
\end{aligned}
$$

Substituting expressions (9) and (10) in expressions (6) and (7), we get

$$
\begin{gathered}
A_{r+1, z}+A_{r-1, z}+A_{r, z+1}+A_{r, z-1}-\left(4-\frac{h_{r}+h_{z}}{2 i}\right) A_{r, z}-4 \frac{h_{r} h_{z}}{v} J=0 \\
A_{r+1, z}+A_{r-1, z}+A_{r, z+1}+A_{r, z-1}-\left(4-\frac{h_{r}+h_{z}}{2 i}\right) A_{r, z}-4 \frac{h_{r} h_{z} H_{C}}{v}=0 \\
A_{r+1, z}+A_{r-1, z}+A_{r, z+1}+A_{r, z-1}-\left(4-\frac{h_{r}+h_{z}}{2 i}\right) A_{r, z}=0
\end{gathered}
$$

Expressions (11-13) are used to calculate the magnetic potential in the internal nodes of the generator.

The nodes lying in the zone of the magnetizing coil are determined from expression (11), the nodes lying in the zone of the permanent magnet are determined from expression (12), taking into account the magnetic potential of the coercive force of the magnet, the nodes lying in the zone of the magnetic pipeline and the air space are calculated from expression (13).

The design of a synchronous generator is such that it contains the separation boundaries of magnetizing and non-magnetizing media, that is, it contains the boundaries separating surfaces with different magnetic permittivity.

Consider a synchronous generator node containing a magnetizing substance (permanent magnet) and a non-conducting medium.

Assume that point 3 belongs to a permanent magnet, which magnetic potential corresponds to the coercive force of the permanent magnet, point 1 belongs to a de -energized region (air). 2 and 4 belong to both regions and are located on the media section. Boundary conditions of the first kind give equality of magnetic potentials located at the interface, that is $A_{a}=A_{b}$. From the condition of continuity of the first derivative, when the magnetic flux lines of force passing through the interface equality of the normal components follow $\partial A / \partial n$ for both areas, the result is: 


$$
v_{a} \frac{\partial A_{a}}{\partial n}=v_{b} \frac{\partial A_{b}}{\partial n}
$$

Approximating expression (14), we get

$$
v_{a}\left(A_{a 1}-A_{a 3}\right)=v_{b}\left(A_{b 1}-A_{b 3}\right)
$$

Given expression (15) and the node configuration shown in Figure 2, we get

$$
\begin{gathered}
2 v_{b} A_{b 1}+\left(v_{a}+v_{b}\right) A_{2}+2 v_{a} A_{a 3}+\left(v_{a}+v_{b}\right) A_{4}+ \\
+\left(v_{a}+v_{b}\right)\left(\frac{h_{r}+h_{z}}{2 i}-4\right) A_{0}-4 \frac{h_{r} h_{z} H_{C}}{\left(v_{a}+v_{b}\right)}=0 \\
2 v_{b} A_{b 1}+\left(v_{a}+v_{b}\right) A_{2}+2 v_{a} A_{a 3}+\left(v_{a}+v_{b}\right) A_{4}+ \\
+\left(v_{a}+v_{b}\right)\left(\frac{h_{r}+h_{z}}{2 i}-4\right) A_{0}=0
\end{gathered}
$$

Formula (16) is used for calculating magnetic and non-conducting surfaces, (17) for calculating only non-conducting surfaces.

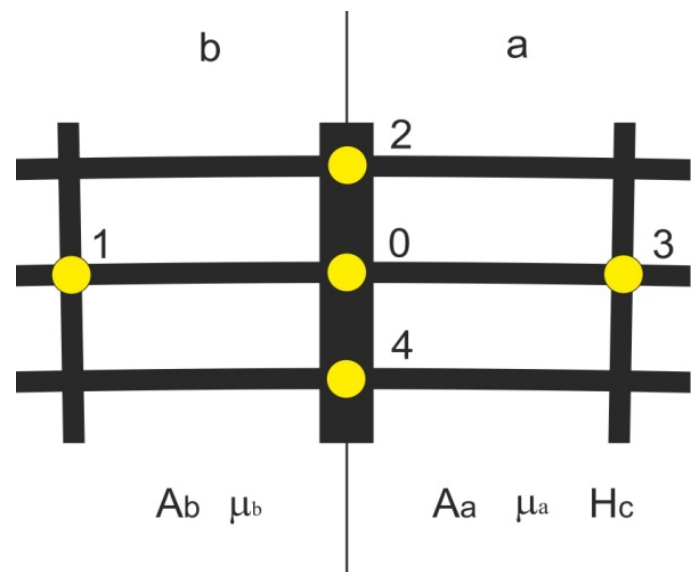

Fig. 2. Node lying on a section of two media: a magnet and a non- conducting part

When calculating magnetic fields using expressions (16) and (17), it is necessary to determine the boundaries of the separation of media, which does not allow to unify this calculation, since the configuration of the magnetic system of the synchronous generator and its elements may change. In this regard, to unify the calculation of any magnetic system, it is necessary to determine mathematical dependencies that allow for the separation of media with different magnetic resistance.

To do this, we improve the method of finite differences. For an explanation, we give the same node as in Figure 2, taking into account the magnetic resistance of the nodes that are connected to the points.

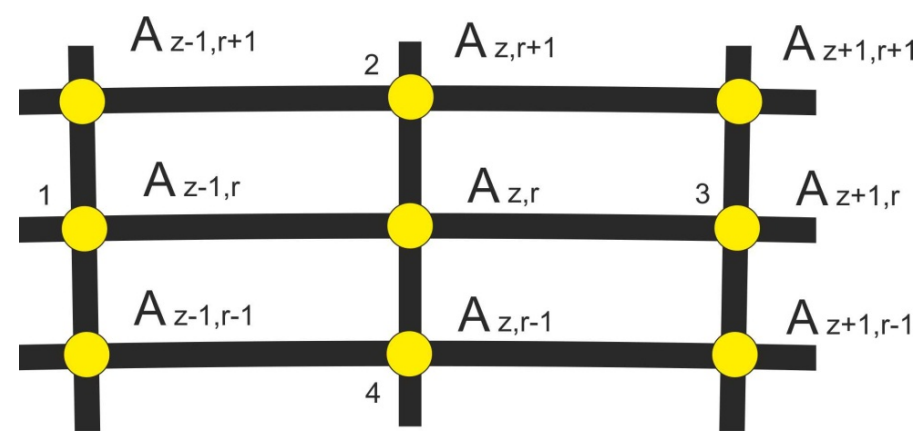

Fig. 3. Node for calculating the magnetic potential taking into account the separation of media 
Calculation of the magnetic potential at the point $A_{z, r}$ is calculated based on the magnetic potentials at the points $A_{z-1, r} A_{z+1, r} A_{z, r-1} A_{z, r+1}$. To determine whether these points are located on the media section, the magnetic resistance of the points associated with them is taken into account.

For example, for the point $A_{z+1, r}$ the magnetic resistance of points is determined $A_{z-1, r+1} A_{z+1, r+1}$, if it is the same, then the point $A_{z+1, r}$ it is not located on the media section, if it is different, then the average magnetic resistance of the points is applied for this point $A_{z-1, r+1} A_{z+1, r+1}$.

The calculation is performed in the same way, for example, for the point $A_{z-1, r}$ the magnetic resistance of points is determined $A_{z-1, r+1} A_{z+1, r-1}$, if it is the same, then the point $A_{z-1, r}$ it is not located on the media section, if it is different, then the average magnetic resistance of the points is applied for this point $A_{z-1, r+1} A_{z-1, r+1}$.

Thus, it is possible to unify the calculation of any axisymmetric magnetic system, accounting for the media partition in which it is made, using the magnetic resistances of conjugate points to the points of the calculation node.

Thus, taking into account expressions (11-13) and the proposed improvement of the finite difference method for calculating the magnetic field of a synchronous generator, we obtain expressions (18-20) for calculating the magnetic potential at a point.

Expression (18) is used to calculate the magnetic potential of current-carrying surfaces, expression (19) is used to calculate the magnetic potential of permanent magnets, and expression (20) is used to calculate the magnetic potential of non-current-carrying parts.

Based on the obtained expressions (18-20), we will perform mathematical modeling of the magnetic potential distribution in the sector of a synchronous generator with a two-circuit magnetic system.

Denoted $\Sigma v_{2}=v_{A_{z-1, r+1}}+v_{A_{z+1, r+1}} ; \Sigma v_{3}=v_{A_{z+1, r+1}}+v_{A_{z+1, r-1}} ; \Sigma v_{4}=v_{A_{z-1, r-1}}+v_{A_{z+1, r-1}}$;

$$
\begin{gathered}
\Sigma v_{1}=v_{A_{z-1, r-1}}+v_{A_{z-1, r+1}} ; \Sigma v=v_{A_{t-1, r+1}}+v_{A_{z+1, r+1}}+v_{A_{z-1, r-1}}+v_{A_{z+1, r-1}} \text { get } \\
A_{z, r}=\frac{\left(A_{z, r+1}\left(\Sigma v_{2}\right)+A_{z+1, r}\left(\Sigma v_{3}\right)+A_{z, r-1}\left(\Sigma v_{4}\right)+A_{z-1, r}\left(\Sigma v_{1}\right)\right)}{\left(\frac{1}{2} \Sigma\left(4-\frac{h_{r}+h_{z}}{2 i}\right)\right)}-\frac{h_{r} h_{z} J}{v_{A_{z, r}}} \\
A_{z, r}=\frac{\left(A_{z, r+1}\left(\Sigma v_{2}\right)+A_{z+1, r}\left(\Sigma v_{3}\right)+A_{z, r-1}\left(\Sigma v_{4}\right)+A_{z-1, r}\left(\Sigma v_{1}\right)\right)}{\left(\frac{1}{2} \Sigma v\left(4-\frac{h_{r}+h_{z}}{2 i}\right)\right)}-\frac{h_{r} h_{z} H_{C}}{v_{A_{z, r}}} \\
A_{z, r}=\frac{\left(A_{z, r+1}\left(\Sigma v_{2}\right)+A_{z+1, r}\left(\Sigma v_{3}\right)+A_{z, r-1}\left(\Sigma v_{4}\right)+A_{z-1, r}\left(\Sigma v_{1}\right)\right)}{\left(\frac{1}{2} \Sigma v\left(4-\frac{h_{r}+h_{z}}{2 i}\right)\right)}
\end{gathered}
$$

To do this, we use the following sequence of actions to perform the calculation.

1. The MS Excel program creates a synchronous generator material model with a symbol in each cell.

2. Based on the created map, the following tab in the MS Excel table sets the magnetic permeability for each material.

3. Initial magnetic potentials equal to 0 are set for all cells.

4. The following table sets the values of the coercive force for each cell of the neodymium magnet, the values of the cells of the other materials are assumed to be 0 .

5. In our case, the calculated field has 180 by 48 cells, which determines the necessary number of calculations in the cycle to obtain a picture of the distribution of the magnetic potential. The number of cycles to calculate is 8640 .

6. The magnetic field of the generator is calculated in stationary mode, that is, without current flowing through its winding. Expressions (19) and (20) are used for this purpose. 
7. According to the obtained picture of the distribution of the magnetic potential, the magnetic induction vector in $\mathrm{r}$ and $\mathrm{z}$ is found.

The magnetic induction vector is determined from expressions (20), (21).

$$
\begin{gathered}
B=r o t A \\
B_{z}=\frac{1}{r} \frac{\partial A}{\partial r}, B_{r}=\frac{\partial A}{\partial z}
\end{gathered}
$$

1. We construct an axisymmetric system of the induction vector B.

2. A graph of magnetic induction is plotted along the middle line of the generator winding in the direction of the $\mathrm{R}$ axis.

When modeling the magnetic field distribution of a synchronous generator, the following values were taken: the coercive force of a niodymium magnet of the $\mathrm{N} 40-979000 \mathrm{~A} \cdot \mathrm{m}^{-1}$ brand ; the magnetic permeability of the synchronous generator magnetic core $-2.3 \cdot 10^{-2} \mathrm{GH} \cdot \mathrm{m}^{-1}$; magnetic permeability of air $-1.256 \cdot 10^{-6} \mathrm{GH} \cdot \mathrm{m}^{-1}$; magnetic permeability of copper $-1.256 \cdot 10^{-6} \mathrm{GH} \cdot \mathrm{m}^{-1}$; magnetic permeability of neodymium magnet $-1.32 \cdot 10^{-6} \mathrm{GH} \cdot \mathrm{m}^{-1}$.

Neodymium magnet is a magnetohard material, the value of the magnetic potential at the point is determined by the magnitude of the coercive force. Neighboring nodes have a slight influence on the level of magnetic potential of the calculated node of the neodymium magnet, which is associated with the hysteresis loop of this magnet.

\section{Results and discussion}

Mathematical modeling is performed for the following cases: synchronous generator with an external magnetic circuit, with an internal magnetic circuit, and with two circuits. The magnetic induction vector on the synchronous generator sector plan is shown in the following figures.

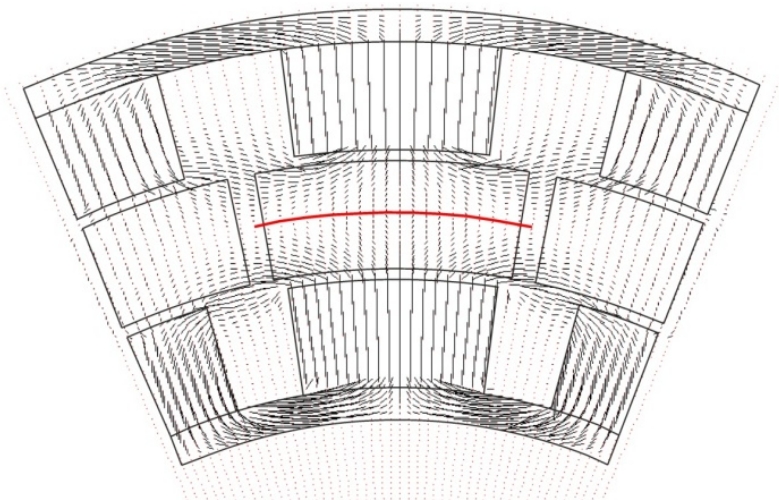

Fig. 4. Magnetic induction vector on the plan of the generator sector with external and internal magnetic circuits

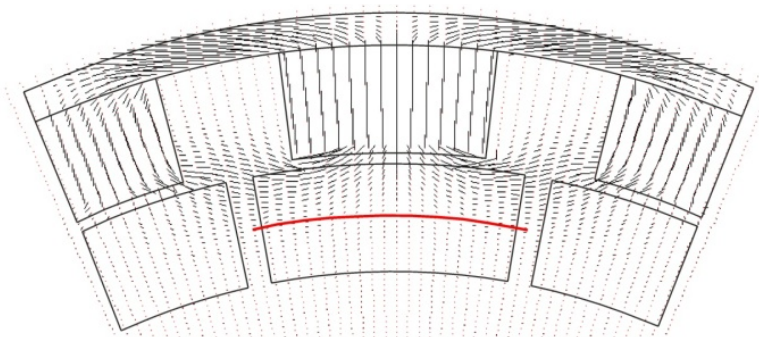

Fig. 5. Magnetic induction vector on the plan of the generator sector with an external magnetic circuit 


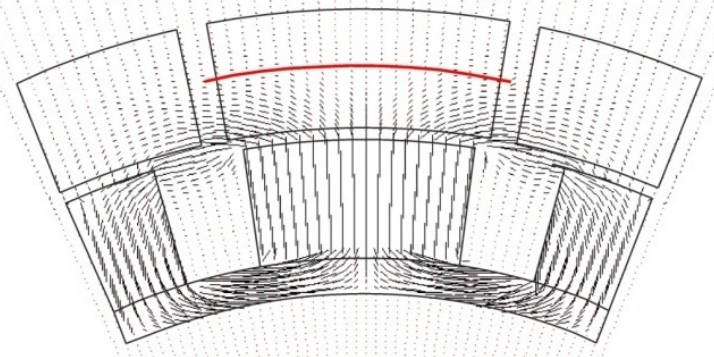

Fig. 6. Magnetic induction vector on the plan of the generator sector with an internal magnetic circuit

Figures 4-6 show the distribution of the magnetic induction vector from the calculated nodes. Let us plot the magnetic induction vector $\mathrm{B}_{\mathrm{r}}$, along the $\mathrm{z}$ axis passing through the middle of the synchronous generator winding, as shown above.

The obtained results of calculating the magnetic field pattern are in good agreement with the results of calculation in the FEMM and ELCUT programs, which indicates a high accuracy of calculation using this method for axisymmetric systems.

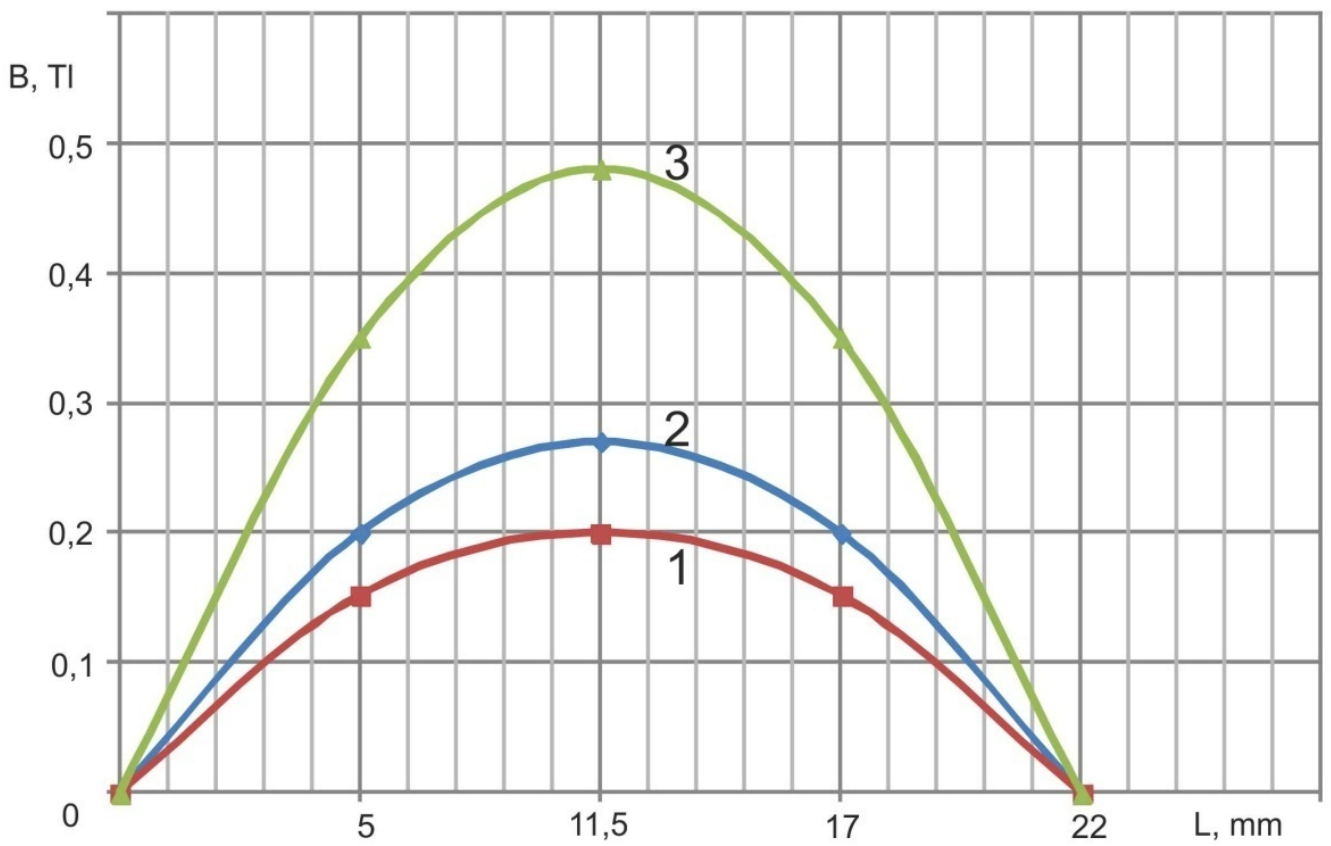

Fig. 7. Graphs of changes in the magnetic induction vector $\mathrm{Br}$ along the length of the generator winding: 1 - two circuits; 2 - external circuit; 3 - internal circuit

\section{Conclusions}

1. Using the proposed method for calculating the magnetic system allows to automate the calculation process by using the proposed method for determining the boundaries of media materials.

2. The mathematical modeling on the example of calculation of the magnetic system of the synchronous generator showed that the maximum value of the magnetic induction $B_{r}$, penetrating the winding of the synchronous generator along the axis $r$, when the internal circuit is $0.21 \mathrm{~T}$, using the external loop, the value of the magnetic induction vector is $0.283 \mathrm{~T}$. When using a twocircuit system, the value of the magnetic induction vector $B_{r}$ is $0.483 \mathrm{~T}$, which proves the 
effectiveness of using a two-circuit magnetic system of a synchronous generator with permanent magnets.

\section{References}

[1] Nikitenko, G., Konoplev, E., Salpagarov, V., Lysakov, A. Solar and wind stand-alone power system. Engineering for Rural Development №18, 2019. pp. 1456-1462.

[2] Pat. 2680642 Russian Federation, IPC H02K 1/27, H02K 21/12, H02K 16/02 Synchronous generator with dual magnetic system/Nikitenko G. V., Konoplev V. E., Salpagarov K. V., Konoplev, P. V., Bobryshev, A. V.; applicant and patent holder FGBOU IN Stavropol GAU. No. 2016152824; application. 30.12.16; publ. 25.01.18, Byul. No. 3.

[3] Andreeva E.G., Tatevosyan A.A. Mathematical Description of Solution of the Three-Dimensional Boundary Value Problem for the Stationary Magnetic Field in the Cylindrical Coordinate System. Journal of Physics: Conference Series. 2020. 1441(1).

[4] Zubkov Y.V., Makarichev Y.A., Antropov V.E. Finding electromagnetic loads and magnetic-field factors in design of integrated brushless excitation DC generator. Proceedings - 2019 International Ural Conference on Electrical Power Engineering, UralCon. 2019. 8877639, pp. 217-222.

[5] Yousefian M., Zarchi H.A., Gorginpour H. Modified steady-state modelling of brushless doublyfed induction generator taking core loss components into account. IET Electric Power Applications, 2019. 13(9), pp. 1402-1412.

[6] Abdalla S.A., Abdullah S.S. Performance improvements of induction motor drive supplied by hybrid wind and storage generation system based on mine blast algorithm. Energies, 2019. 12(15), 2947. 